Supporting Information

\title{
Monitoring the removal efficiency of pharmaceuticals and hormones in different treatment processes of source-separated urine with bioassays
}

\author{
Beate I. Escher* \\ Wouter Pronk \\ Marc J.-F. Suter \\ Max Maurer
}

Swiss Federal Institute of Aquatic Science and Technology (Eawag),

$\mathrm{CH}-8600$ Dübendorf, Switzerland

**Corresponding author phone: 0041-44-823 5068; fax: 0041-44-823 5471;

e-mail: escher@eawag.ch. 


\section{Pharmaceuticals spiked to urine}

The pharmaceuticals carbamazepine (CAS RN 298-46-4, no purity reported), diclofenac (CAS RN 15307-856-5, no purity reported), 17 $\beta$-estradiol (E2, CAS RN 50-28-2), $17 \alpha-$ ethinylestradiol (EE2, CAS RN 57-63-6, >98\%), ibuprofen (CAS RN 15687-27-1, >98\%), propranolol (CAS RN 525-66-6, >98\%), sulfamethoxazole (CAS RN 723-46-6, no purity reported) were obtained from Sigma (Buchs, Switzerland). All solvents and salts were obtained from Fluka (Buchs, Switzerland).

The concentrations of the pharmaceuticals spiked to urine were chosen according to toxic potency and predicted urine concentration (PUC). The PUC can be computed with equation S-1 (adapted from PEC calculation proposed by EMEA (1)) from the amount A $(\mathrm{kg})$ sold per year in a country, the number of inhabitants $P$ per country, the molecular weight $\mathrm{MW}(\mathrm{kg} / \mathrm{mol})$ of the compound and the produced urine per capita per day $U(\mathrm{~L} / \mathrm{d})$. We assume that approx. $1 \mathrm{~L}$ of urine is excreted per capita per day. The excretion rate $E R$ is the fraction of unmetabolized pharmaceutical in urine. We computed two scenarios, PUC(1), where ER was set to 1 (worst case scenario), and PUC(2), where ER was calculated from the fraction excreted unchanged and directly conjugated in the urine (realistic case).

$\mathrm{PUC}(\mathrm{M})=\frac{\mathrm{A} \cdot \mathrm{ER}}{\mathrm{P} \cdot \mathrm{U} \cdot 365 \mathrm{~d} / \mathrm{y} \cdot \mathrm{MW}}$

The concentrations spiked to urine were 1.3 to 16 times higher than the PUC, reflecting that not just seven compounds but a much larger number of chemicals are present in urine. Also, in order not to have a too small contribution to the overall effect by the $\beta$ blocker propranolol, its concentration was increased by a factor of 250 over the PUC. This choice is justified because propranolol constitutes only $1.6 \%$ of the total sales of $ß$ blockers in Germany in 2001 (2).

\section{Endogenous compounds in urine}

Urine contains a multitude of endogenous compounds of lower hydrophobicity, typically $\log \mathrm{K}_{\mathrm{ow}}<2.1$ (3). In Table S-1B, the nine most hydrophobic compounds in urine are listed and their concentration in urine. Despite the relatively low hydrophobicity, these compounds may well contribute to the non-specific toxicity because the concentrations are in the high micromolar concentration range, adding up to a millimolar total concentration.

\begin{tabular}{lcr}
\hline Natural urine components & $\log \mathrm{K}_{\text {ow }}$ & concentration in urine $(\mathrm{M})$ \\
\hline 4-Hydroxyindol & 1.57 & $7.83 \cdot 10^{-4}$ \\
Rhodanid & 0.58 & $5.83 \cdot 10^{-5}$ \\
Phenol & 1.46 & $9.17 \cdot 10^{-5}$ \\
p-Cresol & 1.94 & $6.67 \cdot 10^{-4}$ \\
Brenzcatechin & 0.88 & $4.17 \cdot 10^{-5}$ \\
Phenylacetic acid & 1.49 & $9.75 \cdot 10^{-4}$ \\
p-Hydroxybenzoic acid & 1.58 & $1.75 \cdot 10^{-4}$ \\
p-Hydroxyphenylacetic acid & 0.75 & $2.92 \cdot 10^{-4}$ \\
m-Hydroxyphenylacetic acid & 1.23 & $6.67 \cdot 10^{-5}$ \\
Sum of concentrations (M) & & $3.15 \cdot 10^{-3}$ \\
\hline
\end{tabular}

Table S-1B Natural urine components (selection from (3)). Kow values taken from the the Physical Properties Database (PHYSPROP), accessible at http://www.syr res.com/esc/physprop.htm. 
Table S-1A Physicochemical properties of the mixture of pharmaceuticals used to spike the urine and concentration of the different drug spiked to urine. ${ }^{a}$ Physicochemical data were compiled from literature and $E C_{50}$ were determined in ref. (4). ${ }^{b}$ Data from (5). ${ }^{c-e} P U C$ computed with the sales in Germany in 2001 (data from $\left.{ }^{c}(6),{ }^{d}(7)\right)$, and $\left.{ }^{e}(2)\right)$ and a German population of 82 mio. $^{f}$ Data compiled from (8-10).

\begin{tabular}{|c|c|c|c|c|c|c|c|c|c|c|}
\hline & \multirow{2}{*}{$\begin{array}{c}\text { Octanol- } \\
\text { water } \\
\text { partition } \\
\text { coefficient } \\
\log \mathrm{K}_{\text {ow }}\end{array}$} & $\begin{array}{c}\text { Liposome- } \\
\text { water } \\
\text { distribution } \\
\text { ratio at } \mathrm{pH} 7\end{array}$ & $\begin{array}{l}\text { Acidity } \\
\text { constant }\end{array}$ & $\begin{array}{l}\text { Inhibition } \\
\text { of PSII } \\
\text { quantum } \\
\text { yield }\end{array}$ & $\begin{array}{l}\text { Yeast } \\
\text { estrogen } \\
\text { screen }\end{array}$ & \multicolumn{2}{|c|}{$\begin{array}{l}\text { Concentration in spiked } \\
\text { urine }\end{array}$} & \multicolumn{2}{|c|}{$\begin{array}{c}\text { Predicted urine } \\
\text { concentrations PUC }\end{array}$} & \multirow[b]{2}{*}{$(\mathrm{PUC}(2)$} \\
\hline & & $\begin{array}{l}\log D_{\text {lipw }} \\
\text { at } \mathrm{pH} 7\end{array}$ & $\mathrm{pK}_{\mathrm{a}}$ & $\begin{array}{c}\log (1 / \\
\left.\mathrm{EC}_{50}(\mathrm{M})\right)\end{array}$ & $\begin{array}{c}\log (1 / \\
\left.\mathrm{EC}_{50}(\mathrm{M})\right)\end{array}$ & $\begin{array}{l}\text { Conc. } \\
(\mathrm{M})\end{array}$ & $\begin{array}{l}\text { Conc. } \\
\left(\mathrm{mg} \mathrm{L}^{-1}\right)\end{array}$ & PUC(1) & $E R^{f}$ & \\
\hline Carbamazepine $^{a}$ & 2.45 & 2.73 & - & $<3.00$ & $<3.00$ & $2.0 \cdot 10^{-4}$ & 47.3 & $1.24 \cdot 10^{-5 c}$ & $8 \%$ & $9.91 \cdot 10^{-7}$ \\
\hline Diclofenac $^{a}$ & 4.51 & 2.65 & 3.99 & 3.30 & $<2.00$ & $1.29 \cdot 10^{-4}$ & 38.2 & $9.68 \cdot 10^{-6 c}$ & $21 \%$ & $2.03 \cdot 10^{-6}$ \\
\hline Estradiol & $4.01^{b}$ & & & & & $7.35 \cdot 10^{-9}$ & 0.0020 & & & \\
\hline Ethinyl estradiol ${ }^{a}$ & 3.67 & 3.81 & - & 4.38 & 9.6 & $6.75 \cdot 10^{-9}$ & 0.0020 & $5.35 \cdot 10^{-9 d}$ & $47 \%$ & $2.52 \cdot 10^{-9}$ \\
\hline Ibuprofen $^{a}$ & 3.97 & 1.91 & 4.45 & 3.35 & $<2.00$ & $1.34 \cdot 10^{-4}$ & 24.7 & $6.25 \cdot 10^{-5 c}$ & $21 \%$ & $1.31 \cdot 10^{-5}$ \\
\hline Propranolol $^{a}$ & 3.48 & 2.77 & 9.24 & 5.61 & $<2.00$ & $5 \cdot 10^{-5}$ & 13.0 & $1.93 \cdot 10^{-7 e}$ & $29 \%$ & $5.60 \cdot 10^{-8}$ \\
\hline Sulfamethoxazole ${ }^{a}$ & 0.89 & 1.30 & 5.7 & 3.08 & $<3.00$ & $10^{-5}$ & 2.5 & $7.0710^{-6 c}$ & $35 \%$ & $2.47 \cdot 10^{-6}$ \\
\hline
\end{tabular}




\section{Detailed results from the bioassays}

\section{Electrodialysis}

Table S-2 $E C_{50}$-values in the YES and treatment efficiencies in the electrodialysis experiments. ${ }^{a}$ Equation 9.

\begin{tabular}{lcccccc}
\hline & urine (1) & urine (2) & diluate (1) & diluate (2) & concentrate (1) & concentrate (2) \\
\hline EC $_{50}$ (REF) & 0.005 & 0.003 & 0.007 & 0.008 & 0.72 & n.d. \\
EEEQ (M) & $6.05 \cdot 10^{-8}$ & $8.59 \cdot 10^{-8}$ & $3.97 \cdot 10^{-8}$ & $3.58 \cdot 10^{-8}$ & $4.03 \cdot 10^{-8}$ & $<$ LOD \\
Recovery EEQ (\%) & $88 \%$ & $125 \%$ & $58 \%$ & $52 \%$ & $0.6 \%$ & $0.0 \%$ \\
$\begin{array}{l}\text { Dilution by } \\
\text { electrodialysis process }\end{array}$ & & & & & & \\
Removal efficiency & & & $66 \%$ & $42 \%$ & & \\
\hline
\end{tabular}

\section{Nanofiltration}

Table S-3 $E C_{50}$-values in the YES and treatment efficiencies in the nanofiltration experiments. ${ }^{a}$ Equation 11.

\begin{tabular}{|c|c|c|c|c|c|c|}
\hline & $\begin{array}{c}\text { retentate } \\
(1)\end{array}$ & $\begin{array}{c}\text { permeate } \\
(1)\end{array}$ & $\begin{array}{c}\text { retentate } \\
(2)\end{array}$ & $\begin{array}{c}\text { permeate } \\
(2)\end{array}$ & $\begin{array}{c}\text { retentate } \\
\text { (3) }\end{array}$ & $\begin{array}{c}\text { permeate } \\
\text { (3) }\end{array}$ \\
\hline $\begin{array}{l}\mathrm{EC}_{50}(\mathrm{REF}) \\
\text { Relative removal }\end{array}$ & 0.006 & 0.013 & 0.002 & 0.003 & 0.004 & 0.010 \\
\hline efficiency $(\%)^{a}$ & & $53.3 \%$ & & $47.6 \%$ & & $59.4 \%$ \\
\hline
\end{tabular}

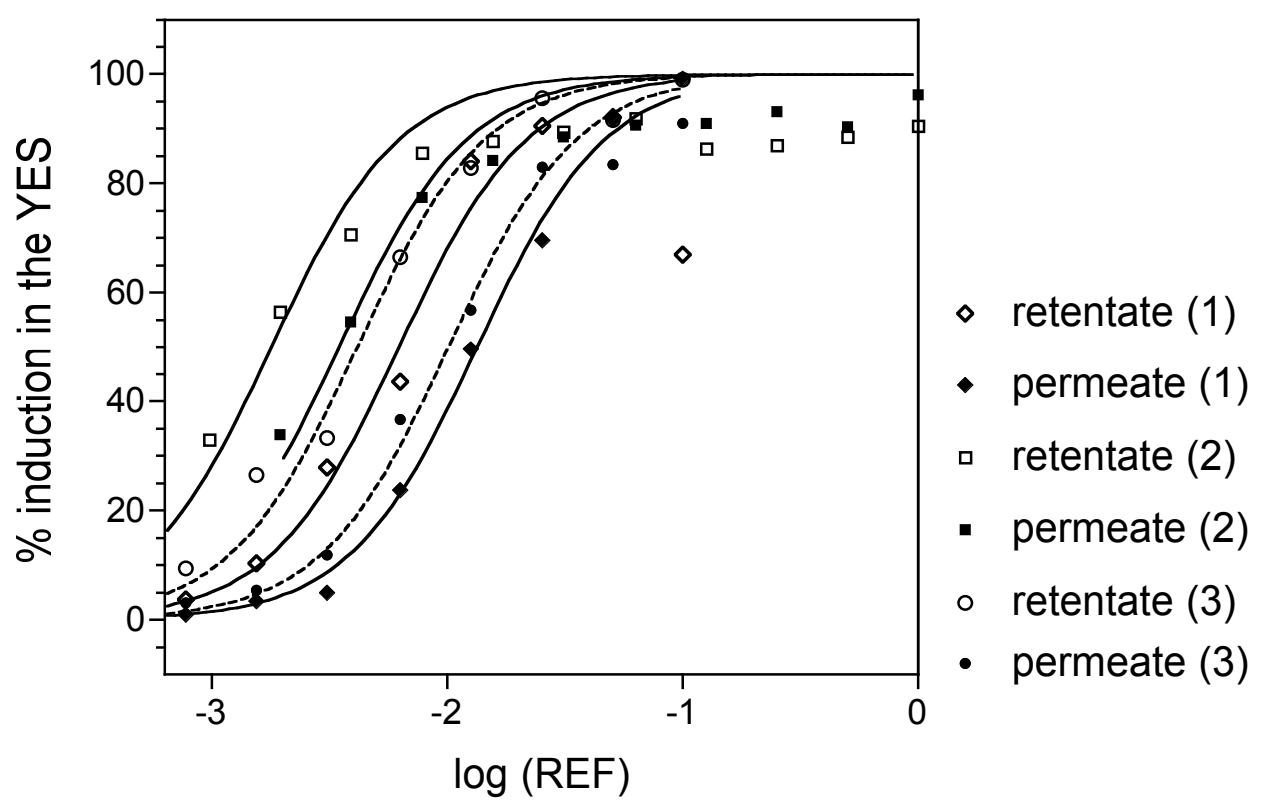

FIGURE S-1. Concentration-effect curve in the YES after nanofiltration. (1), (2) and (3) refer to three independent experiments, retentate refers to the retentate after nanofiltration, permeate refers to the permeate after nanofiltration. 
Table S-4 EC $C_{50}$-values in the algal toxicity test and treatment efficiencies in the nanofiltration experiments. ${ }^{a}$ Equation 9. ${ }^{b}$ Equation 11.

\begin{tabular}{lccr}
\hline & urine (1) & $\begin{array}{c}\text { urine + mixture of } \\
\text { pharmaceuticals (2) }\end{array}$ & $\begin{array}{c}\text { urine + mixture of } \\
\text { pharmaceuticals (3) }\end{array}$ \\
\hline EC $_{50}(\mathrm{REF})$ before nanofiltration & 0.18 & 0.09 & 0.10 \\
$\mathrm{EC}_{50}(\mathrm{REF})$ retentate & 0.19 & 0.10 & 1.1 \\
$\mathrm{EC}_{50}(\mathrm{REF})$ permeate & 1.1 & 0.73 & 0.84 \\
Removal efficiency (\%) $^{\mathrm{a}}$ & $84 \%$ & $88 \%$ & $89 \%$ \\
Relative removal efficiency (\%) $^{\mathrm{b}}$ & $91 \%$ & $74 \%$ & $88 \%$ \\
\hline
\end{tabular}

Table S-5 Concentrations in nanofiltration experiment (2) (see Table S-3 for corresponding algal toxicity data). ${ }^{a}$ Equation 9. ${ }^{b}$ Equation 11.

\begin{tabular}{|c|c|c|c|c|}
\hline & propranolol & ibuprofen & diclofenac & carbamazepine \\
\hline $\begin{array}{l}\text { Concentration }(\mu \mathrm{M}) \text { before } \\
\text { nanofiltration }\end{array}$ & 47 & 143 & 139 & 198 \\
\hline Concentration $(\mu \mathrm{M})$ retentate & 52 & 169 & 149 & 217 \\
\hline Concentration $(\mu \mathrm{M})$ permeate & 19 & 5.6 & 4.9 & 52 \\
\hline Removal efficiency $(\%)^{a}$ & $59 \%$ & $96 \%$ & $96 \%$ & $74 \%$ \\
\hline Relative removal efficiency $(\%)^{b}$ & $63 \%$ & $97 \%$ & $97 \%$ & $77 \%$ \\
\hline
\end{tabular}

\section{Struvite precipitation}

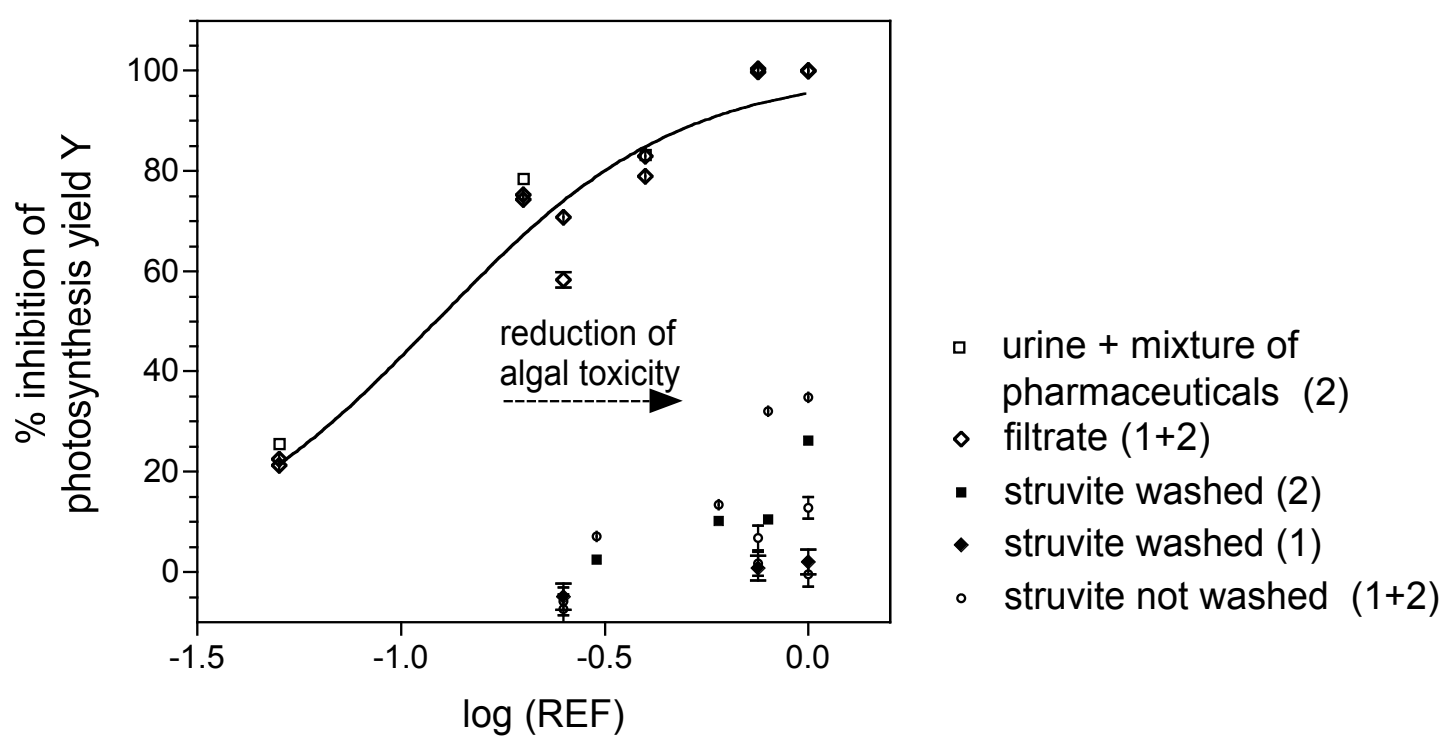

FIGURE S-3. Concentration-effect curve in the algal chlorophyll fluorescence test after struvite. (1) and (2) refer to two independent experiments, filtrate and start solutions overlapped and concentration-effect curves of filtrates from both experiments were therefore fitted together. Struvite refers to redissolved struvite, washed or not washed before dissolution. 
Table S-6 $E C_{50}$-values in the algal chlorophyll fluorescence assay and treatment efficiencies in the struvite precipitation experiment. ${ }^{a}$ Equation $9 .{ }^{b}$ Equation 11.

\begin{tabular}{|c|c|c|c|c|c|}
\hline & $\begin{array}{l}\text { urine }+ \\
\text { mixture of } \\
\text { pharma- } \\
\text { ceuticals }(1+2)\end{array}$ & $\begin{array}{l}\text { filtrate } \\
(1+2)\end{array}$ & $\begin{array}{l}\text { struvite } \\
\text { washed (1) }\end{array}$ & $\begin{array}{l}\text { struvite } \\
\text { washed (2) }\end{array}$ & $\begin{array}{r}\text { struvite not } \\
\text { washed } \\
(1+2)\end{array}$ \\
\hline $\mathrm{EC}_{50}(\mathrm{REF})$ & 0.10 & 0.13 & n.d. & 2.5 & 3.0 \\
\hline $\begin{array}{l}\text { Removal efficiency }(\%)^{a} \\
\text { Relative removal efficiency in } \\
\text { relation to filtrate }(\%)^{b}\end{array}$ & & & $\begin{array}{l}>99 \% \\
>99 \%\end{array}$ & $\begin{array}{l}96.1 \% \\
95.1 \%\end{array}$ & $\begin{array}{l}96.7 \% \\
95.9 \%\end{array}$ \\
\hline
\end{tabular}

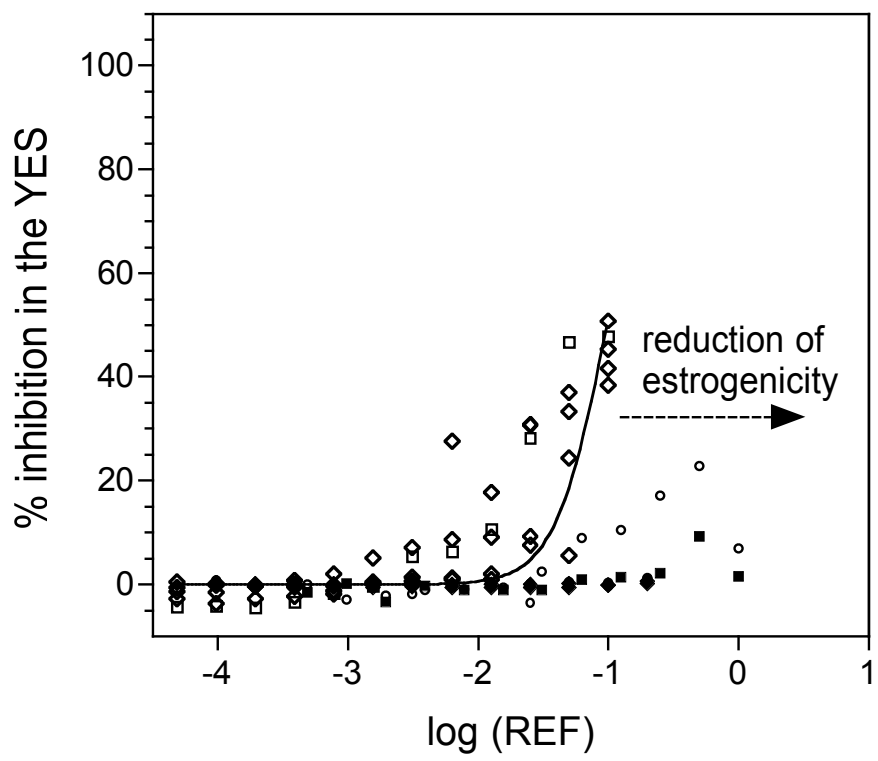

a $\quad$ urine + mixture of pharmaceuticals (2)

$\diamond$ filtrate $(1+2)$

- struvite washed (2)

- struvite washed (1)

- struvite not washed $(1+2)$

FIGURE S-4. Concentration-effect curve in the YES test after struvite precipitation. (1) and (2) refer to two independent experiments, filtrate and start solutions overlapped and concentration-effect curves of filtrates from both experiments were therefore fitted together. Struvite refers to redissolved struvite, washed or not washed before dissolution.

Table S-7 EC $C_{50}$-values in the YES and treatment efficiencies in the struvite precipitation experiment. ${ }^{a}$ Equation 9. ${ }^{b}$ Equation 11.

\begin{tabular}{|c|c|c|c|c|c|}
\hline & $\begin{array}{l}\text { urine }+ \\
\text { pharma- } \\
\text { cocktail }(1+2)\end{array}$ & $\begin{array}{l}\text { filtrate } \\
(1+2)\end{array}$ & $\begin{array}{l}\text { struvite } \\
\text { washed (1) }\end{array}$ & $\begin{array}{l}\text { struvite } \\
\text { washed (2) }\end{array}$ & $\begin{array}{r}\text { struvite not } \\
\text { washed } \\
(1+2)\end{array}$ \\
\hline $\mathrm{EC}_{50}(\mathrm{REF})$ & 0.07 & 0.10 & n.d. & 4.4 & 2.3 \\
\hline $\begin{array}{l}\text { Removal efficiency }(\%)^{a} \\
\text { Relative removal efficiency in } \\
\text { relation to filtrate }(\%)^{b}\end{array}$ & & & $\begin{array}{l}97.2 \% \\
96.0 \%\end{array}$ & $\begin{array}{l}98.5 \% \\
97.8 \%\end{array}$ & $\begin{array}{l}97.1 \% \\
95.8 \%\end{array}$ \\
\hline
\end{tabular}




\section{Ozonation}

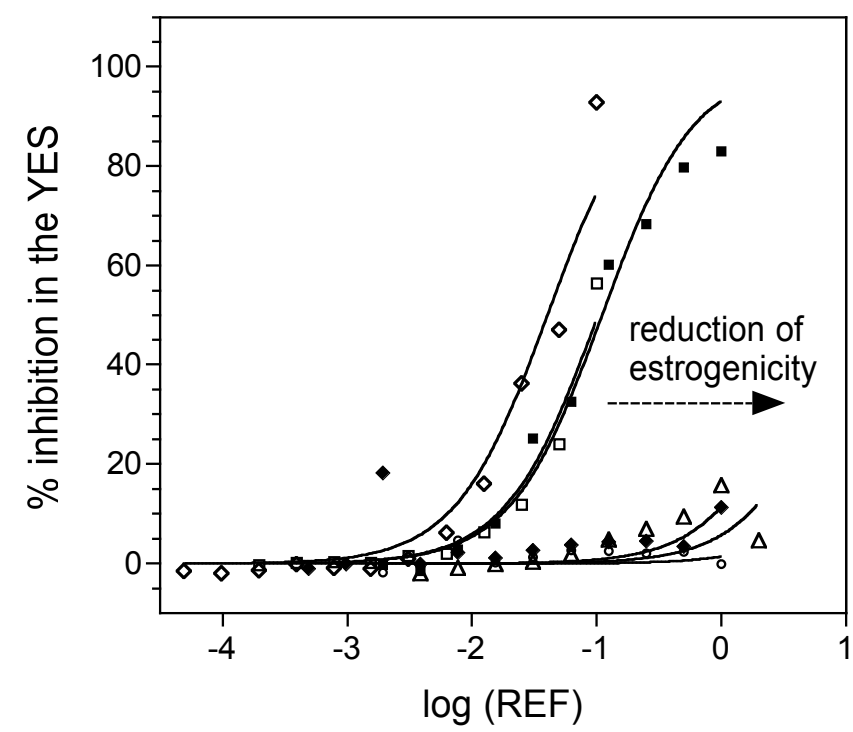

$\diamond$ urine + mixture of pharmaceuticals (1)

- urine + mixture of pharmaceuticals (2)

- after ozonation $0.7 \mathrm{gO}_{3} / \mathrm{L}$ (1)

- after ozonation $0.57 \mathrm{gO}_{3} / \mathrm{L} \mathrm{(2)}$

- after ozonation $1.1 \mathrm{gO}_{3} / \mathrm{L}(2)$

$\Delta \quad$ after ozonation $2.1 \mathrm{gO}_{3} / \mathrm{L}(2)$

FIGURE S-5A. Concentration-effect curve in the YES test during ozonation experiment. (1) and (2) refer to two independent experiments.



- urine + mixture of pharmaceuticals (2)

- after ozonation $0.57 \mathrm{gO}_{3} / \mathrm{L}(2)$

- after ozonation $1.1 \mathrm{gO}_{3} / \mathrm{L} \mathrm{(2)}$

$\Delta$ after ozonation $2.1 \mathrm{gO}_{3} / \mathrm{L} \mathrm{(2)}$

FIGURE S-5B. Concentration-effect curve in the Anti-YES test during ozonation experiment. In each well 3.1 $10^{-10}$ M E2 was added. (2) refers to the same experiment as in Figure S-5A, some data points at higher concentrations could not be evaluated due to growth inhibitory effects of the yeast cells. The line(s) correspond to the effect level of the added E2. 
Table S-8. $E C_{50}$-values in YES test and treatment efficiencies during the ozonation experiments. (1) and (2) refer to two independent experiments. ${ }^{a}$ Equation 9.

\begin{tabular}{|c|c|c|c|c|c|c|}
\hline & $\begin{array}{l}\text { urine }+ \\
\text { mixture of } \\
\text { pharma- } \\
\text { ceuticals (1) }\end{array}$ & $\begin{array}{l}\text { urine }+ \\
\text { mixture of } \\
\text { pharma- } \\
\text { ceuticals (2) }\end{array}$ & $\begin{array}{l}\text { after } \\
\text { ozonation } \\
0.7 \mathrm{gO}_{3} / \mathrm{L} \\
\text { (1) }\end{array}$ & $\begin{array}{l}\text { after } \\
\text { ozonation } \\
0.57 \mathrm{gO}_{3} / \mathrm{L} \\
\text { (2) }\end{array}$ & $\begin{array}{l}\text { after } \\
\text { ozonation } \\
1.1 \mathrm{gO}_{3} / \mathrm{L} \\
(2)\end{array}$ & $\begin{array}{r}\text { after } \\
\text { ozonation } \\
2.1 \mathrm{gO}_{3} / \mathrm{L} \\
\text { (2) }\end{array}$ \\
\hline $\mathrm{EC}_{50}(\mathrm{REF})$ & 0.04 & 0.10 & 5.58 & 0.11 & 35.0 & 10.5 \\
\hline Removal efficiency (\%) & & & $99.3 \%$ & $7.1 \%$ & $99.7 \%$ & $99.0 \%$ \\
\hline
\end{tabular}

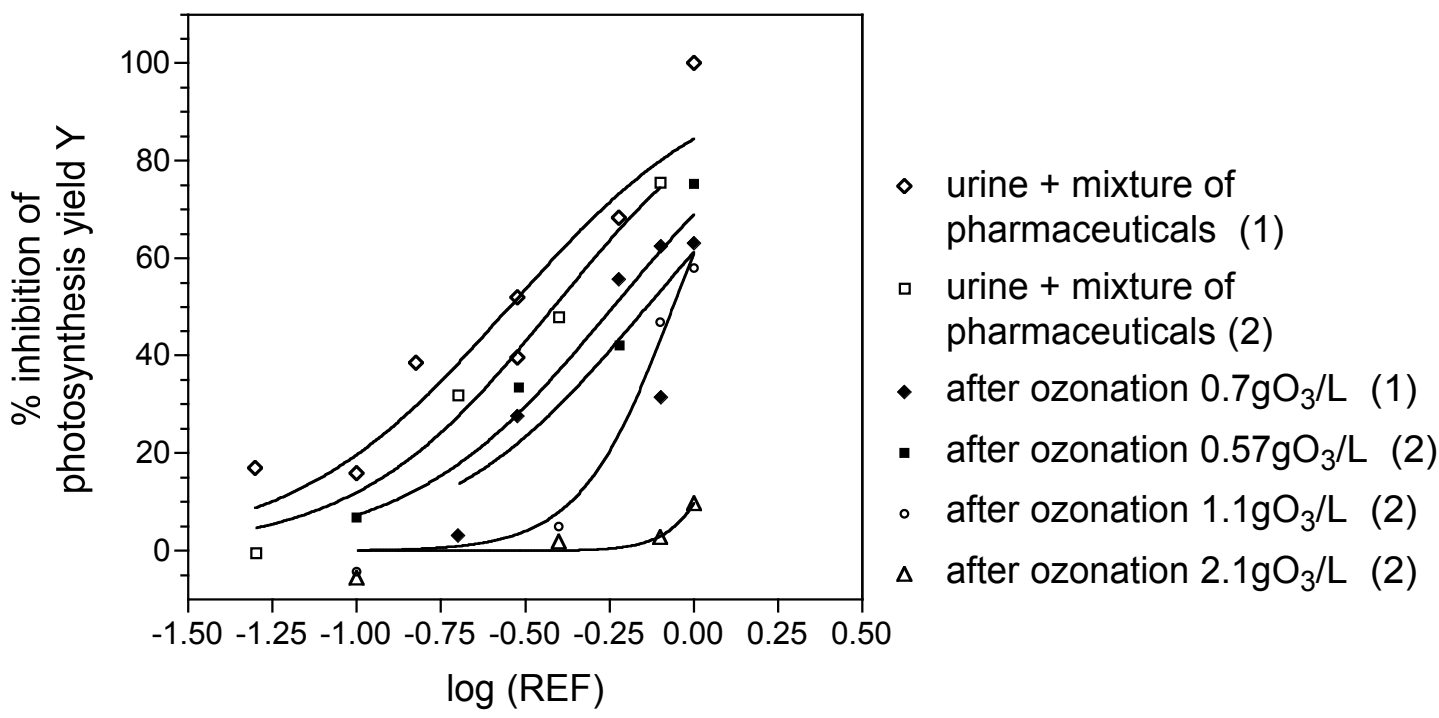

FIGURE S-6. Concentration-effect curve in the algal chlorophyll fluorescence test during ozonation experiment. (1) and (2) refer to two independent experiments.

Table S-9 $E C_{50}$-values in the algal chlorophyll fluorescence assay and treatment efficiencies in the ozonation experiments. (1) and (2) refer to two independent experiments. ${ }^{2}$ Equation 9.

\begin{tabular}{|c|c|c|c|c|c|c|}
\hline & $\begin{array}{l}\text { urine }+ \\
\text { mixture of } \\
\text { pharma- } \\
\text { ceuticals (1) }\end{array}$ & $\begin{array}{l}\text { urine }+ \\
\text { mixture of } \\
\text { pharma- } \\
\text { ceuticals (2) }\end{array}$ & $\begin{array}{l}\text { after } \\
\text { ozonation } \\
0.7 \mathrm{gO}_{3} / \mathrm{L} \\
\text { (1) }\end{array}$ & $\begin{array}{l}\text { after } \\
\text { Ozonation } \\
0.57 \mathrm{gO}_{3} / \mathrm{L} \\
(2)\end{array}$ & $\begin{array}{l}\text { after } \\
\text { ozonation } \\
1.1 \mathrm{gO}_{3} / \mathrm{L} \\
(2)\end{array}$ & $\begin{array}{r}\text { after } \\
\text { ozonation } \\
2.1 \mathrm{gO}_{3} / \mathrm{L} \\
(2)\end{array}$ \\
\hline $\mathrm{EC}_{50}(\mathrm{REF})$ & 0.28 & 0.38 & 0.73 & 0.58 & 0.87 & 1.5 \\
\hline Removal efficiency $(\%)^{a}$ & & & $60.8 \%$ & $33.3 \%$ & $55.7 \%$ & $74.5 \%$ \\
\hline
\end{tabular}




\section{References}

(1) EMEA "Draft Guideline on the Environmental Risk Assessment of Medicinal Products for Human Use CHMP/SWP/4447/00," The European Agency for the Evaluation of Medicinal Products, 2005.

http://www.emea.eu.int/pdfs/human/swp/444700en.pdf.

(2) Cleuvers, M., Initial risk assessment for three beta-blockers found in the aquatic environment. Chemosphere 2005, 59, 199-205.

(3) Ciba Geigy Harn- Physikalisch-chemische Daten. In Wissenschaftliche Tabellen Geigy-Körperflüssigkeiten; Ciba Geigy AG, Ed.: Basel, Switzerland, 1977; pp 51-96.

(4) Escher, B. I.; Bramaz, N.; Eggen, R. I. L.; Richter, M., In-vitro assessment of modes of toxic action of pharmaceuticals in aquatic life. Environ. Sci. Technol. 2005, 39, 3090-3100.

(5) Hansch, C.; Leo, A.; Hoekman, D. Exploring QSAR. Hydrophobic, Electronic and Steric Constants; American Chemical Society: Washington, DC, 1995.

(6) Huschek, G.; Hansen, P. D.; Maurer, H. H.; Krengel, D.; Kayser, A., Environmental risk assessment of medicinal products for human use according to the European Commission recommendations. Environ. Toxicol. 2004, 226-240.

(7) Liebig, M.; Moltmann, J. F.; Knacker, T., Evaluation of measured and predicted environmental concentrations of selected human pharmaceuticals and personal care products. Environ. Sci. Poll. Res. 2006, 13, 110-119.

(8) Saller, R. Praktische Pharmakologie. Eigenschaften gebräuchlicher Medikamente; Schattauer: Stuttgart, Germany, 1983.

(9) Dollery, C.; Boobis, A. R.; Burley, D.; Davies, D. M.; Davies, D. S.; Harrison, P. I.; Orme, M. L. E.; Park, B. K.; Goldberg, L. I., Eds. Therapeutic drugs; Churchill Livingstone: New York, NY, 1991.

(10) Baselt, R. C.; Cravey, R. H. Disposition of Toxic Drugs and Chemicals in Man; fourth edition; Chemical Toxicology Institute: Foster City, CA, USA, 2000. 